\title{
Entendendo o Uso de Design Thinking no Apoio ao Desenvolvimento de Software à luz das Areas de IHC e Sistemas Colaborativos
}

\author{
Lauriane Correa \\ MunDDoS - PPGCC \\ Escola Politécnica, PUCRS \\ Porto Alegre - RS \\ lauriane.moraes@acad.pucrs.br
}

\author{
Sabrina Marczak \\ MunDDoS - PPGCC \\ Escola Politécnica, PUCRS \\ Porto Alegre - RS \\ sabrina.marczak@pucrs.br
}

\author{
Tayana Conte \\ USES - PPGI \\ Inst. de Computação, UFAM \\ Manaus - AM \\ tayana@icomp.ufam.edu.br
}

\begin{abstract}
RESUMO
O Design Thinking aparece na Engenharia de Software como uma abordagem promissora, incentivando a colaboração, auxiliando no entendimento e consequente especificação das necessidades do usuário. Inspirado em práticas de Design centradas no usuário, a abordagem ainda tem seus benefícios pouco conhecidos no apoio ao desenvolvimento de software. Assim, este projeto visa identificar como o Design Thinking tem sido usado neste contexto. Propõe-se uma abordagem empírica de pesquisa baseada em múltiplos estudos com usuários e profissionais da indústria, bem como especialistas no uso da abordagem para explorar o assunto. Estes estudos serão conduzidos à luz de conceitos das areas de Interação Humano-Computador (e.g., design centrado no usuário, personas) e Sistemas Colaborativos (e.g., colaboração), vislumbrando contribuir para a integração das áreas. Um primeiro estudo está em andamento com instrutores e alunos de academias de musculação visando tratar a problemática de instrutores e alunos trabalharem distantes uns dos outros e em relação aos treinos definidos. Resultados preliminares do estudo indicam que o Design Thinking de fato permite uma melhor compreensão do usuário e suas necessidades. Estudos futuros fazem parte da nossa proposta de pesquisa para corroborar e expandir estes insights iniciais.
\end{abstract}

\section{Palavras-chave Autor}

Desenvolvimento de Software; Colaboração em Equipes de Software; Design Thinking; IHC; Estudo Emp'irico.

\section{Classificação Palavras-chave ACM}

H.5.m. Information Interfaces and Presentation (e.g. HCI): Miscellaneous

\section{INTRODUÇÃO}

A indústria de software tornou-se altamente competitiva, havendo vários produtos do mesmo domínio de aplicações

Permission to make digital or hard copies of all or part of this work for personal or classroom use is granted without fee provided that copies are not made or distributed for profit or commercial advantage and that copies bear this notice and the full citation on the first page. Copyrights for components of this work owned by others than the author(s) must be honored. Abstracting with credit is permitted. To copy otherwise, or republish, to post on servers or to redistribute to lists, requires prior specific permission and/or a fee. Copyright 2018 SBC.

IHC 2018, Anais Estendidos do XVII Simpósio Brasileiro sobre Fatores Humanos em Sistemas Computacionais

Outubro 22-26, 2018, Belém, Brasil

Fórum de Integração e Discussão sobre Interação Humano-Computador e Sistemas Colaborativos (FID IHC-SC) para atender aos usuários. Um produto precisa destacarseperante os similares, satisfazendo seus usuários com carac- terísticas novas e úteis [2]. Os problemas que soluções de software têm tratado estão também cada vez mais complexos, exigindo equipes multidisciplinares e a proximidade com os envolvidos na questão-os usuários, para auxiliar no seu en- tendimento e proposta de solução.

O Design Thinking (DT) surge como um conjunto de práticas inspiradas no Design para resolução e desenvolvimento de problemas, utilizando a empatia, a criatividade e a racionalidade para atender às necessidades dos usuários e concretizar os objetivos [20]. Está sendo utilizado para facilitar a geração de diferentes soluções para um determinado problema, pois combina o foco no usuário final, colaboração multidisciplinar e melhorias iterativas na criação de artefatos [14].

A abordagem ganhou popularidade por ser vista como um novo paradigma para lidar com a resolução de problemas em áreas como Educação e Medicina [7], e não foi diferente na área de Tecnologia da Informação, em especial no desenvolvimento de software. A inspiração se deve também pela busca de profissionais com intuito de aproximar usuários e desenvolvedores. O desenvolvimento ágil foi um passo neste sentido, mas estudos apontam que empresas de software ainda continuam a desenvolver produtos que não atendem às expectativas dos usuários [1].

Assim, este projeto visa identificar como a abordagem tem sido usada no apoio ao desenvolvimento de software (e.g., práticas de uso, melhorias percebidas pela colaboração promovida, melhoria da qualidade dos requisitos em função do uso integrado de técnicas de engenharia e Design, etc). Considera-se a investigação do uso desta abordagem pertinente a esta trilha de integração do Interação HumanoComputador (IHC) com Sistemas Colaborativos (SC) visto a natureza intr' inseca colaborativa do desenvolvimento de software, trazendo aspectos da área de SC, e a origem do DT ser baseado em princ'ipios de Design e ter seu foco centrado no usuário, os quais tem suas raízes na área de IHC. Assim, este artigo brevemente apresenta o conceito de DT ediscorre sobre seu uso no desenvolvimento de software, bem como introduz a visão geral da investigação proposta, incluindo sua abordagem metodológica e destacando um estudo inicial em realização. Também discorre sobre como este tópico tem potencial para integrar não apenas pesquisadores mas também as áreas de IHC e SC no contexto de desenvolvimento de software. 


\section{DESIGN THINKING}

O termo Design Thinking normalmente éatribu'ido a Peter Rowe, por sua publicação intitulada 'Design Thinking', em 1987, sobre o processo de Design na arquitetura e no planejamento urbano [15]. Desde então diversos modelos de Design Thinking (DT) emergiram, com base na utilização em diferentes teorias das áreas de Design, Psicologia e Educação [7]. DT é uma abordagem centrada no usuário, composta de um conjunto de técnicas e ferramentas, que utiliza uma visão iterativa para produzir e analisar de forma criativa soluções para desafios reais [16]. É vista como uma forma eficaz no descobrimento de informações detalhadas, visando a empatia dos usuários e entendimento do contexto de uso para desenvolver uma maior compreensão da necessidade destes usuários [20]. Sucintamente, Brown (2008) destaca três pontos importantes [4]: (i) foca no Design centrado no ser humano, (ii) permite a criação de soluções de projetos viáveis que atendam às necessidades dos clientes com valor agregado; e (iii) serve para projetar artefatos, portanto, Design éparte integrante do DT.

Dentre os modelos de DT, o modelo de processo de Design duplo diamante (Double Diamond Design Process model) é um dos mais conhecidos [6]. O modelo éconstitu'ido de 4 etapas, denominadas: Descobrir, Definir, Desenvolver e Entregar, organizadas em estágios divergentes e convergentes do processo de Design, conforme ilustrado na Figura 1. Mais especificamente, tem-se: (1) Primeira etapa, primeiro diamante, representa a parte inicial divergente do projeto: a etapa de descoberta, na qual o designer procura novas oportunidades, mercados, informações, tendências e insights [18]. Também chamada de imersão preliminar, pois objetiva o entendimento inicial do problema [8]. (2) Segunda etapa, primeiro diamante, marca o estágio de definição, um tipo de 'filtro' no qual os primeiros insights são revisados e selecionados [18]. Também chamada de imersão em profundidade, pois identifica as necessidades e oportunidades para a geração de soluções nas próximas etapas [8]. (3) Terceira, segundo diamante, representa o per'́odo de desenvolvimento de potenciais soluções para a etapa anterior [18]. Também chamada de análise e síntese, pois os envolvidos usam todos os dados obtidos, agrupando os que são em comum. Isto faz com que os problemas mais recorrentes sejam identificados [8]. E, (4) Quarta etapa, segundo diamante, visa a convergência da solução e marca a entrega, que por sua vez centra todas as etapas com a validação do que foi produzido [18].

Embora haja diversas definições para DT, todas gravitam na mesma filosofia que abrange o pensamento criativo, o conhecimento contextual e os cenários na aplicação de uma possível solução [13]. Para isto, diversas técnicas são utilizadas, como destacado a seguir. A (a) Pesquisa de Campo Inicial visa a obtenção da compreensão do contexto, auxiliando no entendimento de perfis de usuários e do ambiente em que a equipe explorará a possível solução do problema. Estas pesquisas objetivam o entendimento geral do contexto e suas tendências [8]. (b) A Pesquisa desk objetiva fazer levantamentos nas mais variadas fontes sobre o projeto e suas tendências. Esta pesquisa é feita quando existe a necessidade de aprofundar informações a respeito de questões sugeridas ao longo do projeto. Também chamada de pesquisa

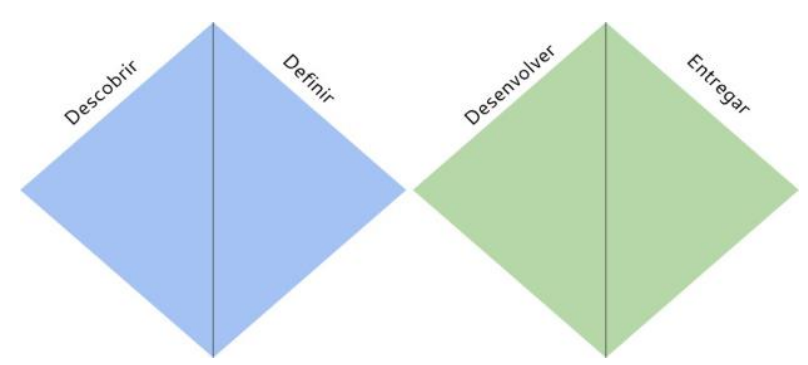

Figure 1. Modelo Duplo Diamante [6].

secundária e consiste na equipe tentar reunir o máximo de informações sobre o contexto. Estas são capturadas de meios como internet, jornais, TV, livros, entre outros [8]. (c) $\mathrm{O}$ Brainstorming reúne pessoas que se concentram em discutir um determinado assunto, proporcionando uma dinâmica em grupo para coletar informações e ideias sob diversas perspectivas [12]. O objetivo égarantir o debate de diversas ideias para escolha futura baseada em critérios de seleção como desejo do usuário, viabilidade tecnológica e de negócios [3]. (d) A técnica Persona consiste na elaboração de uma pessoa fict'icia que sintetiza os comportamentos, representando as motivações, desejos, expectativas e necessidades dos envolvidos no uso da solução [5]. Já a (e) Jornada do usuário descreve os passos percorridos antes, durante e depois de determinada atividade do usuário a ser analisada [10].

Os estudos sobre DT no apoio ao desenvolvimento de software são ainda limitados. Por exemplo, Souza, Ferreira e Conte (2017) [17] identificaram através de um mapeamento sistemático da literatura que as técnicas e ferramentas utilizadas para apoiar o uso de DT neste contexto são em geral definidas baseadas no tipo de problema a ser resolvido, no público-alvo, no tempo de projeto, e entre outros fatores relacionados ao contexto do problema [17]. Por sua vez, Vetelli et al. (2013) [19] argumentaram que a utilização de DT pode auxiliar os processos de engenharia de requisitos visto que DT incentiva a identificação das necessidades dos clientes, mas não demonstraram a aplicabilidade de sua proposição.

\section{OBJETIVO DA PESQUISA}

Como um primeiro passo para o entendimento de como o Design Thinking (DT) pode apoiar o desenvolvimento de software, esta pesquisa visa primeiramente caracterizar como a abordagem DT tem sido utilizada neste contexto. Mais especificamente, visa-se explorar este fenômeno à luz dos conceitos das área de IHC e Sistemas Colaborativos com o intuito de promover a integração das área.

Vai-se seguir uma abordagem emp'irica qualitativa [9] no desenvolvimento da pesquisa visando a aproximação com profissionais da indústria e usuários bem como especialistas no uso de DT para facilitar o entendimento do fenômeno na prática. A pesquisa vai ser realizada baseada em múltiplos estudos, quais sejam: um estudo com usuários para identificar a aplicabilidade do uso de DT na prática (em andamento, concluindo a etapa de coleta e análise de dados, com resultados preliminares a serem apresentados [11]), um estudo de campo baseado em entrevistas com profissionais da indústria 
para identificar como os mesmos têm usado DT em seu dia a dia (planejamento em andamento, com mais de 50 profissionais da indústria confirmados como participantes candidatos), e uma survey para corroborar o aprendizado da etapa anterior.

O primeiro estudo aplicou 10 técnicas propostas pelo DT com instrutores e alunos de academias de musculação para o entendimento de como ambos papéis podem se aproximar e visar a qualificação dos treinos dos alunos. A situação problemática foi escolhida baseada na experiência pessoal de dois dos autores, sendo um deles é Bacharel em Educação Física. Como resultados das etapas Descobrir e Definir do modelo Duplo Diamante [6], identificou-se que o DT de fato promove a interação com o usuário e o engajamento dos mesmos.

\section{INTERSECÇÃO COM IHC}

O tema de Design Thinking inerentemente utiliza-se de conceitos da área de IHC bem como promove a colaboração com usuários, o que caracteriza um dos aspectos investigados pela área de Sistemas Colaborativos. Este tema vai ser explorado no contexto de desenvolvimento de software, que por sua vez apresenta intrinsecamente caracter'1sticas colaborativas. Desta forma, esta pesquisa aproxima ambas as área. Os estudos vão seguir uma abordagem empírica qualitativa visando o entendimento do fenômeno na prática, visando, também, identificar as potenciais técnicas que promovem integração entre IHC e SC. Estes estudos vão ser conduzidos por uma pesquisadora em fase de doutoramento com experiência na indústria (primeira autora) e duas pesquisadoras seniores que possuem seus interesses de pesquisa nas área de SC (segunda autora) e IHC (terceira autora) bem como a Engenharia de Software como área de interseção. Ambas pesquisadoras seniores possuem histórico de trabalharem conjuntamente em projetos emp' iricos e qualitativos, e participam ativamente das área mencionadas, tendo sido a oportunidade de integração das área a razão principal da parceria estabelecida.

\section{Agradecimentos}

As autoras agradecem o apoio financeiro do $\mathrm{CNPq}$ (processos440880/2013-0, 310468/2014-0, 423149/2016-4 e 311494/2017-0) e da CAPES (processo 175956/2013).

\section{REFERENCES}

1. Ardito, C., Buono, P., Caivano, D., Costabile, M. F., Lanzilotti, R., Ardito, C., Buono, P., Caivano, D., Costabile, M., and Lanzilotti, R. Investigating and promoting ux practice in industry: An experimental study. International Journal of Human - Computer Studies 72, 6 (2014), 542-551.

2. Bhowmik, T., Niu, N., Savolainen, J., and Mahmoud, A. Leveraging topic modeling and part-of-speech tagging to support combinational creativity in requirements engineering. Requirements Engineering 20, 3 (2015), 253-280.

3. Brenner, W., and Uebernickel, F. Design Thinking for Innovation: Research and Practice. Springer Cham Heidelberg New York Dordrecht London, 2016.

4. Brown, T. Design thinking. $84-92$.
5. Cooper, A. The Origin of Personas. Cooper Jornal, 2003.

6. Design Council, D. The $4 \mathrm{~d}$ model or 'double diamond' design process model, 2012.

7. Dorst, K. The core of 'design thinking' and its application. Design Studies 32, 6 (2011), 521 -532.

8. e Silva, M. J. V., e Silva Filho, Y. V., Adler, I. K., de Figueiredo Lucena, B., and Russo, B. Design thinking: inovação em negócios. MJV Press, 2012.

9. Easterbrook, S., Singer, J., Storey, M.-A., and Damian, D. Selecting Empirical Methods for Software Engineering Research. Springer, London, 2008, 285311.

10. Kalbach, J. Mapping Experiences: A Complete Guide to Creating Value Through Journeys, Blueprints, and Diagrams. O'Reilly Media, 2016.

11. Lauriane Correa, Daniel Maria, J. C. B. S. M. T. C. Ao uso de design thinking no apoio ao desenvolvimento de software: Um estudo de caso no contexto de academias de musculação. In Proceedings of the Workshop on Requirements Engineering, Rio de Janeiro, Brazil (To appear (2018), 1-14.

12. Lethbridge, T. C., Sim, S. E., and Singer, J. Studying software engineers: Data collection techniques for software field studies. Empirical Software Engineering 10, 3 (2005), 311-341.

13. Liikkanen, L. A., Laakso, M., and Björklund, T. Foundations for studying creative design practices. In Proc. of the Conference on Creativity and Innovation in Design, ACM, Eindhoven, Netherlands (2011), 309-315.

14. Plattner, H., Meinel, C., and Weinberg, U. Design Thinking: Innovation lernen - Ideenwelten öffnen. mi-Wirtschaftsbuch, 2009.

15. Rowe, P.Design Thinking. MIT Press, 1987.

16. Soledade, M. P., Freitas, R. S., Peres, S. M., Fantinato, M., Steinbeck, R., and Araújo, U. F. Experimenting with design thinking in requirements refinement for a learning management system. 182-193.

17. Souza, A., Ferreira, B. M., and Conte, T. Aplicando design thinking em engenharia de software: Um mapeamento sistemático. In Proceedings of the Iberoamerican Conference on Software Engineering, Buenos Aires, Argentina (2017), 719-732.

18. Tschimmel, K. Design thinking as an effective toolkit for innovation. In Proceedings of the ISPIM Conference: Action for Innovation from Experience (Barcelona, 2012), 1-20.

19. Vetterli, C., Brenner, W., Uebernickel, F., and Petrie, C. From palaces to yurts: Why requirements engineering needs design thinking. IEEE Internet Computing 17, 2 (2013), 91-94.

20. Weigel, L. Design thinking to bridge research and concept design. Wiley, New York, 2015, 59—-70. 